\title{
A series identity
}

By G. N. WATSon, University of Birmingham.

I have constructed two simple proofs of the result established by J. C. P. Miller, Mathematical Notes, No. 29 (1935), pp. vi-viii.

Miller's result may be stated in the following form:

Let

and write

$$
A_{0}=1, \quad A_{n}=\left(-\frac{1}{2} \cdot \frac{1}{4} \ldots \cdot \frac{2 n-3}{2 n}\right)^{2}, \quad(n>0)
$$

$$
S_{1}=\sum_{n=0}^{\infty} \frac{A_{n}}{2 x+2 n}, \quad S_{2}=\sum_{n=0}^{\infty} \frac{A_{n}}{2 x-2 n+1} .
$$

Then

$$
S_{1}=S_{2}
$$

whenever $x$ is a positive integer.

Taking $x$ to be unrestricted, combine like terms of $S_{1}$ and $S_{2}$; we get

$$
\begin{aligned}
& S_{1}-S_{2}=\sum_{n=0}^{\infty} \frac{A_{n}(1-4 n)}{(2 x+2 n)(2 x-2 n+1)}
\end{aligned}
$$

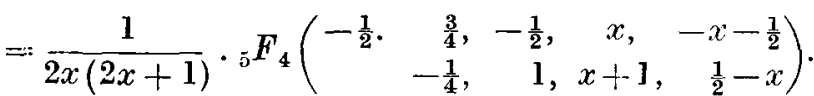

Now, by a theorem due to Dougall,

$$
\begin{aligned}
{ }_{5} F_{4} & \left(\begin{array}{cc}
a, & 1+\frac{1}{2} a, \\
\frac{1}{2} a, & 1+a-c, 1+a-d, 1+a-e
\end{array}\right) \\
& \frac{\Gamma(1+a-c) \Gamma(1+a-d) \Gamma(1+a-e) \Gamma(1+a-c-d-e)}{\Gamma(1+a) \Gamma(1+a-d-e) \Gamma(1+a-e-c) \Gamma(1+a-c-d)} .
\end{aligned}
$$

In Dougall's theorem write

$$
a=c=-\frac{1}{2}, \quad d=x, \quad e=-x-\frac{1}{2},
$$

and we find at once that

$$
\begin{aligned}
S_{1}-S_{2}= & \frac{1}{2 x(2 x+1)} \frac{\Gamma(1) \Gamma\left(\frac{1}{2}-x\right) \Gamma(1+x) \Gamma\left(\frac{1}{2}\right) \Gamma(1) \Gamma\left(\frac{3}{2}+x\right) \Gamma(1-x)}{\Gamma(1-x)}, \\
\text { i.e. } & S_{1}-S_{2}=-\frac{\Gamma(x) \Gamma\left(-x-\frac{1}{2}\right)}{8 \Gamma\left(\frac{3}{2}+x\right) \Gamma(1-x)},
\end{aligned}
$$

whence Miller's result is obvious. A proof of Dougall's theorem is to be found in W. N. Bailey's Generalised hypergeometric series (Cambridge, 1935), pp. 27-28, with complete references to the relevant literature. 
The result given above for unrestricted $x$ suggests my second proof, which is considerably simpler. From the asymptotic expansion of the Gamma-function it follows that the function

$$
-\frac{\Gamma(x) \Gamma\left(-x-\frac{1}{2}\right)}{8 \Gamma\left(\frac{3}{2}+x\right) \Gamma(1-x)}
$$

satisfies the conditions (Cf. Modern Analysis, $\S 7 \cdot 4$ ) which admit of its being expressed as a sum of partial fractions. Since the residues of the function at the poles $x=-n, x=n-\frac{1}{2}$ are respectively

we obtain anew the result

$$
\frac{1}{2} A_{n}, \quad-\frac{1}{2} A_{n},
$$

$$
-\frac{\Gamma(x) \Gamma\left(-x-\frac{1}{2}\right)}{8 \Gamma\left(\frac{3}{2}+x\right) \Gamma(1-x)}=\sum_{n=0}^{\infty} \frac{A_{n}}{2 x+2 n}-\sum_{n=0}^{\infty} \frac{A_{n}}{2 x-2 n+1}
$$

for unrestricted $x$.

It is, of course, possible to write down any number of generalisations of this result, the simplest perhaps being the expression for

$$
\frac{\Gamma(x) \Gamma(-x-a)}{\Gamma(1+a+x) \Gamma(1-x)}
$$

as a sum of partial fractions, where $a$ is a suitably restricted constant.

\section{On the configuration known as a double-six of lines}

By H. W. Richmond, King's College, Cambridge.

In geometry of three dimensions it is well known that, when two quadrics $Q_{1}, Q_{2}$ are given, if one set of four points exists having the properties that each point lies on $Q_{1}$, and each two points are conjugate with respect to $Q_{2}$, an infinity of such sets of points can be found. The quadrics $Q_{1}, Q_{2}$ stand in a special relation to one another ${ }^{1}$, expressed by the vanishing of the coefficient of $\lambda$ in the discriminant of $Q_{2}+\lambda Q_{1}$, an invariant of $Q_{1}, Q_{2}$. Two quadrics $Q_{1}, Q_{2}$ are thus related if the equation of $Q_{1}$ contains no squares

1 See Salmon, Analytic Geometry of Three Dimensions, Rogers' revised edition, Vol. 1, p. 204 ; or Sommerville, Analytical Geometry of Three Dimensions, p. 309. 